\title{
Infection and Bronchiolitis Obliterans Among Native American Lung Transplant Recipients
}

\author{
Amrita Dosanjh $^{*, 1}$ and James Koziol ${ }^{2}$ \\ ${ }^{1}$ UCSD School of Medicine, La Jolla, CA, USA \\ ${ }^{2}$ The Scripps Research Institute, La Jolla, CA, USA
}

\begin{abstract}
Background: Alaska Native and Native American populations (AN/NA) are prone to respiratory tract infections, due to genetic predisposition and environmental factors. Since community acquired respiratory tract infections are associated with higher rates of bronchiolitis obliterans syndrome (BOS), it was hypothesized that AN/NA lung transplant recipients may experience a higher rate of BOS.

Methods: The UNOS database was searched from 1995-2005 to identify adult AN/NA patients undergoing lung transplantation in the U.S. Among 11,103 patients, 33 AN/NA (13M/20F) patients were identified for further analysis. Among this population rates of: i) initial hospital stay, ii) first year hospitalizations, iii) hospitalization for infection, iv) subsequent BOS in years 1-5, were compared. Statistical analysis was performed using the fisher exact test, and a $\mathrm{p}$ value of $<0.05$ was considered significant.

Results: AN/NA recipients did not have a higher incidence of BOS in years 1-5 following lung transplantation. They did have a higher rate of first year complications, reflected by higher hospitalization rates. AN/NA patients had a higher rate of first year hospitalizations for non-CMV infection $(\mathrm{p}<0.03)$.

Conclusions: AN/NA patients despite being at risk for community acquired respiratory infections did not have a higher rate of transplantation BOS in subsequent years.
\end{abstract}

\section{INTRODUCTION}

The Alaskan Native and Native American populations (AN/NA) are prone to respiratory and sinus infections due to a multitude of immunologic, genetic and environmental factors. Native American children are more likely to develop bronchiolitis, asthma and chronic cough [1]. Following pneumonia, Native Americans tend to recover more slowly and have higher rates of chronic cough. Rates of pneumonia and related respiratory illness remain high on Native American reservations [2]. The reasons for the predisposition of native populations to infection may be related to a number of factors, such as crowding, use of wood burning stoves, partial immunizations, and genetic differences in innate and adaptive immunity [3].

The most commonly acquired respiratory infections include influenza A and B, parainfluenza serotypes 1,2, and 3, respiratory syncytial virus ( RSV), adenovirus, rhinovirus, coronaviruses and metapneumovirus [4]. The development of Bronchiolitis Obliterans syndrome, among lung transplant recipients remains a serious complication. The occurrence of BOS is associated with respiratory infection [4].

Since AN/NA patients are prone to respiratory infections, and respiratory infection has been linked to the development of BOS, this population may be at risk for infection and BOS post transplantation. The purpose of this study was to review the incidence of BOS among this population, and to investigate the rate of serious infections, in comparison to a control group.

*Address correspondence to this author at the UCSD School of Medicine, San Diego, CA 92122, USA; Tel: 858442 6146;

E-mail: adosanjh@ucsd.edu

\section{MATERIALS AND METHODOLOGY}

The UNOS database was searched from years 1995-2005 to identify Native American or Alaskan Native (AN/NA) lung transplantation recipients.

Several demographic features were identified and included mean age, age range, gender, and state of origin, for the AN/NA group representing tribal and possible genotypic association. Among the 33 Alaska Native/Native American patients, there were 16 states represented. These regions were representative of major Native American groups. Alaska had the highest number $(n=4)$ of AN/NA lung transplant patients of any state.

The development of Bronchiolitis Obliterans Syndrome (BOS), mean hospital stay after transplantation, first year incidence of serious infection, and incidence of any hospitalization during the first year post transplant and year one acute rejection episodes, were compared between the two patient groups.

In addition, the incidence of BOS in subsequent years 2, 3,4 and 5 was analyzed, comparing the study population (AN/NA) to the control (other). Serious infection was defined as those requiring hospitalization.

\section{Statistical Analysis}

The Fisher Exact test and Chi Square test of association were performed, as appropriate, on all data sets to compare the each condition studied. Only adult patients were included in the analysis due to the small number of pediatric AN/NA patients.

A p-value of $<0.05$ was considered to be a statistically significant difference between the groups. Statistical analysis 
was performed independently by the Biostatistics Division, the Department of Molecular and Experimental Medicine, The Scripps Research Institute, La Jolla, CA.

\section{Results}

\section{AN/NA Patient Characteristics and Demographics}

There were 11,070 non-native American/Alaskan native patients compared to 33 AN/NA patients in the OPTN database for the ten year period between 1995-2005. The mean ages and ranges were comparable and $47.8+/-14.9$ years $(0-$ $78)$ and 48.2+/-15.2 (2-68) years respectively. There were 13 males and 20 females in the native group, whereas the control group was evenly divided between males and females.

\section{Duration of Initial Hospital Stay}

The mean length of hospital stay from transplantation until discharge was $24.1+/-31(1-485)$ days for the control group and 53.6+/- 93 (9-326) days for the AN/NA group.

\section{Hospitalizations in Year One}

There were a total of 7853 adult lung transplant recipients with available data on first year hospitalization rates. Among the control group, 4556 were hospitalized in the first year. AN/NA patients $(n=20)$ were hospitalized for any reason, more frequently in the first year, compared to the control group $(\mathrm{p}=0.03)$.

Among those hospitalized, 1505 or $34 \%$ of the controls were hospitalized for non-cytomegalovirus (CMV) infection.

In comparison, among AN/NA patients, 18/20 (90\%) were hospitalized. Among those hospitalized, 15 or $83 \%$ were hospitalized for infection. AN/NA patients in the first year after lung transplantation had more hospitalizations, and more non-CMV infections requiring hospitalizations than the control group $(\mathrm{p}=0.03)$.

The number of acute rejection episodes during the first year, was not significantly different between the two groups for all age groups, $(p=n s)$, however, UNOS acute rejection data was only required beginning in 2004 .

\section{Incidence of Bronchiolitis Obliterans Syndrome (BOS)}

Based on available data, the overall first year incidence of BOS for the patients studied from 1995 to 2005 was $9 \%$. There were 613 patients in the control group $(n=7388)$ who developed BOS. Among the AN/NA group, two patients of the 16 adult patients developed BOS. Statistically, there was no significant difference between the groups $(\mathrm{p}=\mathrm{ns})$. The odds for occurrence of BOS in the control group was 0.09 and in the AN/NA group 0.125.

\section{Incidence of BOS in Subsequent Years}

\section{$\underline{\text { Years 2-5 }}$}

During the second year post transplantation, among the 5645 control patients, $17.2 \%$ developed BOS. In comparison, 3 patients $(20 \%)$ of the 15 AN/NA patients over 18 years of age developed BOS.

There was no statistically significant difference between the two adult groups in the development of BOS at the end of year 2 .
At the end of year 3, there were 744 of 4361 (17\%) controls who developed BOS. There was one AN/NA patient who had BOS.

Among the AN/NA patients at the end of year $4(n=4)$ one patient $(25 \%)$ carried the diagnosis of BOS. In comparison, there were 668 control patients $(25 \%)$ who developed BOS.

There was no statistical significance in the incidence of BOS between the control and study groups $(\mathrm{p}=\mathrm{ns})$. By the end of year five, 496 of $2448(20 \%)$ adult control patients developed BOS. There were three AN/NA patients, and one had developed BOS. Overall, there were no statistical differences in BOS incidence between Alaskan Native/ Native Americans and control patients, in years one to five. The results of cumulative BOS incidence from years 1-5 are summarized in Table $\mathbf{1}$.

Table 1. BOS Cumulative Incidence Years One to Five ( $p=n s)$

\begin{tabular}{|c|c|c|c|c|c|}
\hline Year & $\mathbf{1}$ & $\mathbf{2}$ & $\mathbf{3}$ & $\mathbf{4}$ & $\mathbf{5}$ \\
\hline \hline AN/NA & 13 & 20 & 25 & 25 & 33 \\
\hline Other & 8 & 17 & 17 & 25 & 20 \\
\hline
\end{tabular}

\section{DISCUSSION}

This study indicates that following lung transplantation, the incidence of BOS in AN/NA populations is not significantly different compared to the control group (other). BOS is a major cause of morbidity and mortality among lung transplant recipients, and despite having a predisposition to respiratory tract and sinus infections, AN/NA patients did not develop BOS more frequently.

This study found that in year one, there is a higher incidence of serious non-CMV infections requiring hospitalizations among the AN/NA population. This finding may lend insight into the connection between BOS and early infection. While the present study cannot comment on the effect of subclinical infection and the development of BOS, it appears that the occurrence of serious infections in the first year in this population did not predispose the AN/NA group to subsequent BOS.

Overall, the year one hospitalization rates, initial hospital stay, and hospitalizations for infections were higher among AN/NA patients undergoing lung transplantation $(\mathrm{p}=0.03)$. Bacterial and viral complications were not the primary cause of first year death among AN/NA recipients. The AN/NA group did not have higher rates of death related viral and bacterial infections during the first year compared to the control group (data not shown). There were no viral related deaths among the AN/NA group.

For patients surviving the first year of transplantation, the cumulative incidence of BOS was not significantly higher. While this study is limited by the small number of AN/NA patients undergoing lung transplantation in the ten year 1995-2005 time period studied, this is a unique data analysis which should lead to future studies. Another limitation of the study is that as with all studies of a particular ethnic group, it relies on self-identification. This study is the first to report the results of lung transplantation among the AN/NA popula- 
tion. The utilization of the OPTN database provides an advantage of inclusion of national data regardless of region. Since there were so few AN/NA lung transplant recipients identified in a ten year period, a national database is needed to complete the analysis. Chart review coordinated among a few centers may yield more information in the future regarding microbial culture results, virology and environmental factors. These parameters are beyond the scope of this study, which does not attempt to identify particular infections. Rather, this study reports that the cumulative incidence of AN/NA BOS is not different from the general transplanted population. Closer surveillance for non-CMV infections, among the AN/NA population in the first year following lung transplantation may be indicated, based on these findings. Newer advances in the diagnosis and treatment of infectious complications of transplantation, will undoubtedly lead to improvements in outcome for this population.

There have been studies linking the occurrence of BOS to acute rejection episodes, and to community acquired infections. One possible mechanism, corroborated by animal models, is that there is initial injury to the epithelium, followed by induction of pro-fibrotic mediators which lead to dysregulated fibrosis in the airway lumen $[5,6]$.

Other studies in humans have described an association between community acquired respiratory infections, and a higher incidence of both acute rejection and chronic allograft dysfunction [7]. One possible explanation as to why the AN/NA population did not develop BOS despite serious first year non-CMV infections, is that risk factors such as acute rejection, gastroesophageal reflux and bacterial airway colonization may be important in the pathophysiology of BOS. These findings also may indicate that chronic sub-clinical states of inflammation and infection may lead to BOS, whereas early isolated episodes of infection may not.

Respiratory infections induce production of alloreactive cytokines, such as IL-1, IL-6, IL-8 and tumor necrosis factor (TNF) and are associated with acute and chronic airway damage $[8,9]$. In one prospective study of 100 lung transplant recipients, 50 had a clinically diagnosed viral infection, and all patients had viral antigen detection, culture and RTPCR. The authors concluded that the patients with viral infection had a higher rate of acute rejection in the three months from baseline, and lower lung function. The lung function decline in the infected group was sustained for a one year period in $66 \%$ and they met the criteria for BOS [4]. In another study of 33 lung transplantation recipients with 40 documented lower respiratory tract infections, a higher grade of BOS was noted, compared to patients with upper respiratory infections [10].

In a retrospective analysis of 122 lung transplant recipients, 10 had been diagnosed with specific respiratory infections, such as respiratory syncytial virus ( $\mathrm{n}=5 / 10$ with $\mathrm{RSV}$ ). Fifty percent of patients surviving respiratory infection developed BOS [11]. Respiratory infections have also been linked to acute allograft rejection, a key risk factor for BOS [12]. In one study of 259 lung transplant recipients, 21 patients had the diagnosis of community acquired respiratory infections, and one third were attributable to RSV. The authors concluded that respiratory infections are a significant risk factor for development and progression of BOS [13].
In this study, among the AN/NA population, known to be at risk for lower respiratory tract infection, first year hospitalization rates for serious non-CMV infection were higher. The study is limited by the lack of database information on the specific etiologies of the non-CMV infections requiring admission. However, based on several studies, RSV appears to be an important cause of serious lower respiratory tract infections requiring hospitalization. CMV infections are generally not linked to BOS [14-17], and therefore not analyzed in this study.

Genetic predisposition of the HLA-DQB10302 type in the native population has been linked to development of bronchiolitis obliterans following viral infection [18, 19]. Other studies indicate that toll-like receptor 4 polymorphisms render an individual susceptible to severe RSV [20]. Some studies cite contributory environmental and socioeconomic factors, such as crowding, use of tobacco and wood burning stoves [21-23]. Prior studies have indicated that the AN/NA population may have selective immune defects. Differential production of IgG and specific IgG after vaccination have been shown among the AN/NA population compared to Caucasians $[24,25]$. The reasons for the predisposition of the native population to infection are varied and provided the basis of studying the development of BOS in native transplant recipients.

\section{CONCLUSIONS}

In conclusion, among the AN/NA patients undergoing lung transplantation, there is no increased risk of BOS, despite a predilection to serious non-CMV infection in the first year. Further multi-center studies should be done to evaluate these findings. Improved viral diagnostic studies, increased surveillance, prevention and treatment of respiratory infections may be warranted in this population, and may provide insight into the pathogenesis of BOS.

\section{ACKNOWLEDGEMENT}

The authors are grateful to Katrina Anderson of UNOS OPTN for providing assistance with the study.

\section{REFERENCES}

[1] Houston, C.S.; Weiler, R.L.; MacKay, R.W. Native children's lung. J. Can. Assoc. Radiol., 1979, 30, 218-219.

[2] Oseasohn, R.; Skipper, B.E.; Tempest, B. Pneumonia in a Navajo community: a two year experience. Am. J. Respir. Crit. Care Med., 1978, 117, 1003-9.

[3] Liu, L.L.; Stout, J.W.; Sullivan, M.; Solet, D.; Shay, D.K.; Grossman, D.C. Asthma and bronchiolitis hospitalizations among American Indian children. Arch. Pediatr. Adoles. Med. 2000, 154, 991-96.

[4] Kumar, D.; Erdman, D.; Keshavjee, S.; Peret, T.; Tellier, R.; Hadjiliadis, D.; Johnson, G.; Ayers, M.; Siegal, D.; Humar, A. Clinical impact of community acquired respiratory viruses on bronchiolitis obliterans after lung transplant. Am. J. Transplant, 2005, 5, 20312036.

[5] Nicod, L. Mechanisms of airway obliteration after lung transplantation. Proc. Natl. Acad. Sci. USA, 2006, 3, 444-449.

[6] Ikonen, T.S.; Brazelton, T.R.; Berry, G.J.; Shorthouse, R.S.; Morris, R.E. Epithelial re-growth is associated with inhibition of obliterative airway disease in orthotopic tracheal allografts in nonimmunosuppressed rats. Transplantation, 2000, 70, 857-63.

[7] Husain, A.N.; Siddiqui, M.T.; Holmes, E.W.; Chandrasekhar, A.J.; McCabe, M.; Radvany, R.; Garrity, E.R. Analysis of risk factors for the development of bronchiolitis obliterans syndrome. Am. J. Resp. Crit. Care Med., 1999, 159, 829-833. 
[8] Vilchez, R.A.; Dauber, J.; Kusne, S. Infectious etiology of bronchiolitis obliterans: the respiratory viruses connection-myth or reality? Am. J. Transplant., 2003, 3, 245-9.

[9] Winter, J.B.; Gouw, A.S.; Groen, M.; Wildevuur, C.; Prop, J. Respiratory viruses aggravate airway damage caused by chronic allograft rejection in rat lung allografts. Transplantation, 1994, 57, 418-422.

[10] Billings, J.L.; Hertz, M.I.; Savik, K.; Wendt, C.N. Respiratory viruses and chronic rejection in lung transplant recipients. J. Heart Lung Transplant., 2002, 21, 559-566.

[11] Palmer, S.M.; Henshaw, N.G.; Howell, S.E. Community respiratory viral infection in adult lung transplant recipients. Chest, 1998, $113,944-950$.

[12] Anderson, D.J.; Jordan, M.C. Viral pneumonia in recipients of solid organ transplants. Semin. Respir. Infect., 1990, 5, 38-40.

[13] Khalifah, A.P.; Hachem, R.R.; Chakinala, M.M.; Schechtman, K.B.; Patterson, G.A.; Schuster, D.P.; Mohanakumar, T.; Trulock, E.P.; Walter, M.J. Respiratory viral infections are a distinct risk factor for bronchiolitis obliterans syndrome and death. Am. J. Resp. Crit. Care Med., 2004, 170, 181-187.

[14] Girgis, R.E.; Tu, I.; Berry, G.J.; Reichenspurner, H.; Valentine, V.G.; Conte, J.V.; Ting, A.; Johnstone, I.; Miller, J.; Robbins, R.C.; Reitz, B.A.; Theodore, J. Risk factors for the development of obliterative bronchiolitis after lung transplantation. J. Heart Lung Tranplant., 1996, 15, 1200-1208.

[15] Sundaresan, S.; Mohanakumar, T.; Smith, M.A.; Trulock, E.P.; Lynch, J.; Phelan, D.; Cooper, J.D.; Patterson, G.A. HLA A locus mismatches and development of antibodies to HLA after lung transplantation correlate with the developmentof antibodies to HLA after lung transplantation correlate with the development of bronchiolitis obliterans syndrome. Transplantation, 1998, 65, 648-653.

[16] Ettinger, N.A.; Bailey, T.C.; Trulock, E.P.; Storch, G.A.; Anderson, D.; Raab, S.; Spitznagel, E.L.; Dresler, C.; Cooper, J.D. Cytomegalovirus infection and pneumonitis: impact after isolated lung transplantation. Am. J. Resp. Crit. Care Med., 1993, 147, 10171023.

[17] Hohlfeld, J.; Niedermeyer, J.; Hamm, H.; Schäfers, H.J.; Wagner, T.O.; Fabel, H. Seasonal onset of bronchiolitis obliterans syndrome in lung transplant recipients. J. Heart Lung Transplant., 1996, 15, 888-894.

[18] Sharples, L.D.; Tamm, M.; McNeil, K.; Higenbottam, T.W.; Stewart, S.; Wallwork, J. Development of bronchiolitis obliterans syndrome in recipients of heart-lung transplantation- early risk factors. Transplantation, 1996, 61, 560-566.

[19] Bridges, N.D.; Spray, T.L.; Collins, M.H.; Bowles, N.E.; Towbin, J.A. Adenovirus infection in the lung results in graft failure after lung transplantation. J. Thorac. Cardiovasc. Surg., 1998, 116, 617623.

[20] Tal, G.; Mandelberg, A.; Dalal, I.; Cesar, K.; Somekh, E.; Tal, A.; Oron, A.; Itskovich, S.; Ballin, A.; Houri, S.; Beigelman, A.; Lider, O.; Rechavi, G.; Amariglio, N. Association between common Tolllike receptor 4 mutations and severe respiratory syncytial virus disease. J. Infect. Dis., 2004, 189, 2057-63.

[21] Dosanjh, A. Adenovirus and Post-Infectious Bronchiolitis Obliterans in Children. Curr. Pediatr. Rev., 2007, 3, 233-237.

[22] Smith, K.J.; Fan, L.L. Insights into post- infectious bronchiolitis obliterans in children. Thorax, 2006, 61, 462-3.

[23] Meissner, H.C. Selected populations at increased risk from respiratory syncytial virus infection. Pediatr. Infect. Dis. J., 2003, 2, S404.

[24] Santosham, M.; Rivin, B.; Wolff, M.; Reid, R.; Newcomer, W.; Letson, G.W.; Almeido-Hill, J.; Thompson, C.; Siber, G.R. Prevention of haemophilus influenza type $\mathrm{b}$ infections in Apache and Navajo children. $J$. Infect. Dis., 1992, 165, S144-51.

[25] Mili, F.; Flanders, W.D.; Boring, J.R.; Destefano, F. The associations of race, cigarette smoking and smoking cessation to measures of the immune system among middle aged- men. Clin. Immunol. Immunopathol., 1991, 59, 187-200.

(C) Dosanjh and Koziol; Licensee Bentham Open.

This is an open access article licensed under the terms of the Creative Commons Attribution Non-Commercial License (http://creativecommons.org/licenses/by$\mathrm{nc} / 3.0 /$ ) which permits unrestricted, non-commercial use, distribution and reproduction in any medium, provided the work is properly cited. 